\title{
Semantic Smart Homes: Towards Knowledge Rich Assisted Living Environments
}

\author{
Liming Chen, Chris Nugent, Maurice Mulvenna, Dewar Finlay and Xin Hong \\ University of Ulster \\ \{l.chen, cd.nugent, md.mulvenna, d.finlay, x.hong\}@ulster.ac.uk
}

\begin{abstract}
The technologies under-pinning smart homes offer promising solutions in the realm of assistive living. At present, there are a number of smart home applications being developed with a raft of technologies that provide fragments of the necessary functionality. Nevertheless, there is currently a major gap between these endeavours and the vision of smart homes in which there are adaptive, personalised and context-aware assistance capabilities. To bridge this divide between practice and aspiration, this paper introduces semantic smart homes - a novel conception whose aim is to move from the current state of the art of smart home technologies to the future infrastructure that is needed to support the full richness of the smart home vision. We present a conceptual system architecture for semantic smart homes and elaborate functions and explore the interplay of constituent components. The paper focuses predominantly on the methodology of semantic modeling, content generation and management. We illustrate the potential of the semantic smart homes metaphor through a number of use scenarios.
\end{abstract}

\section{Introduction}

Recently the provision of health and social care is undergoing a fundamental shift towards the exploitation of technologies to support independent living. These efforts have been driven by the ever growing ageing population and the increasingly over-stretched healthcare resources. Smart Homes (SH) have emerged as a mainstream approach to enable the use of technologies in an individual's living environment to facilitate independent living. Smart homes are augmented environments equipped with sensors, actuators and devices, inhabited by the elderly or disabled and monitored/supported by professionals and health services. The primary impetus for smart homes research and development stems from the personal preferences of people to remain in their own home even if they appreciate that they may be at risk. Additionally, smart homes are able to support user-centred personalised healthcare, thus offering the potential to enhance the quality of life for people at home.

There are currently a number of smart homes [1, 2, 3] in development for the purposes of demonstration as well as for the establishment of real living environments. Researchers are using a multitude of technologies that can provide individual aspects of the functionality required for smart homes. For example, technologies in sensor networks, wearable systems, smart devices and Information \& Communication Technologies (ICT) have all been developed for the capture, communication and analysis of data pertaining to environments, inhabitants and events within smart homes [4, 5, 6, 7]. In the communication layer, open standards and protocols [8, 9] have been developed to address data exchange and compatibility issues among different types of devices and services. In activity tracking, monitoring and recognition, approaches and technologies have been researched and experimented aiming to capture, re-construct and further advise the behaviour of smart home inhabitants $[10,11,12]$.

It may, however, be considered that current endeavours in both technologies and solutions are ad hoc, environment dependent and scenario specific. In most cases, data collected from one sensor 
are used only for one purpose and then discarded. Technological solutions are often developed for well-defined specific cases. It is therefore difficult, if not impossible, for them to be applied in a similar situation, usually requiring substantial re-engineering. At present, large-scale sensory data from sensors, inhabitants, environments and external sources can be captured and collected. Nevertheless, these raw data are too primitive to be processed, used and reused, effectively and intelligently. Though many data processing technologies have been developed, the provisioning and deployment of a generic solution through consolidating these fragmented, disjointed technologies is clumsy. It is also not scalable and feasible in real world situations.

The reason for the aforementioned problems can be associated with the fact that existing $\mathrm{SH}$ technologies and infrastructure are not built upon a commonly agreed smart home data model at both data and application levels, together with an expressive representation. This gives rise to three direct consequences in the development and deployment of SH-based solutions:

- Data heterogeneity hinders seamless exchange, integration and reuse of data.

- Application heterogeneity disallows component (i.e., middleware services) reuse in different application scenarios.

- Without the support of formal data models and expressive representation formalisms, current SH technologies are incapable of dealing with rich metadata and their semantics.

The lack of semantics and inability of data sharing and integration reduce the potential to carry out deep, intelligent data analysis and knowledge discovery from multiple data sources, such as trend discovery, pattern recognition and knowledge-based decision making. This ultimately leads to the difficulty of developing and deploying systematic SH solutions with seamless data integration and advanced high-levels of intelligent capabilities.

As such, there is currently a major gap between these endeavours and the aspiration of what $\mathrm{SH}$ should achieve. To bridge this gap require a vision which embraces technical solutions with a high degree of easy-to-use and seamless automation along with flexibility and scalability in system reconfiguration and deployment, and with adaptation, personalisation and contextawareness in assistance provisioning. In this paper we propose the conception of the Semantic Smart Home (SSH). This concept may be viewed as going beyond current $\mathrm{SH}$ technologies through the creation and management of large-scale, rich semantic information, thus enabling and supporting high-level intelligent capabilities. The cornerstone for the SSH is the ontology-based approach to data modelling for entities in smart homes, including inhabitants, environments, devices and services. Semantic modelling offers realistic solutions to a number of research issues faced by $\mathrm{SH}$ based assisted living such as data interoperability, integration and semantic/knowledge based intelligent decision making support.

The remainder of the paper is organised as follows: Section 2 introduces the SSH concept and related work. Section 3 presents a conceptual architecture for the SSH and Section 4 describes SSH modelling and semantic content management. We outline a semantic enabled paradigm of assistance provisioning and advanced use scenarios in Section 5 and conclude the paper with future research work in section 6 .

\section{Semantic smart homes}

\subsection{The concept}

We define SSH as an extension of the current SH in which data, devices and services are given well-defined meaning. This will better enable the environment, devices, services/applications and 
people (inhabitants and professional carers) to work in cooperation through the extraction of more meaning from the data collected and more appropriate support measures offered to the inhabitant. The essence of a SSH is to have data within and across SH defined and linked in a way that it can be used for more effective discovery, automation, integration and reuse across various applications. Specifically, with semantics and relationships in place we can exploit advanced semantic or Artificial Intelligence (AI) based information processing techniques to provide valueadded data processing capabilities such as data integration, interoperability and high-level decision support within and across SH communities [13, 14]. We envisage that the SSH notion will bring the semantic dimension into SH solutions, enable semantic-based knowledge discovery and intelligent processing as has been witnessed within the general Semantic Web community. This will allow us to ultimately move from the current state of the art of SH technologies to the next generation SH infrastructure that is required to address current shortcomings.

Central to the SSH concept and its realization is semantic data modeling and representation. The rationale associated with this concept is that the more semantics and knowledge the data model can hold and represent, the more capabilities and flexibilities that SSH technologies and applications can achieve in the processing of data.

We contend that ontologies and the Semantic Web infrastructure are the enabling technologies for the realization of SSHs. An ontology is an explicit, shared specification of the various conceptualisations in a problem domain $[15,16]$. It defines commonly agreed data/knowledge structures, i.e., domain concepts, their attributes and the relations between them. In addition it also provides a shared vocabulary for describing these structures. This means that data providers, no matter where they are, can use these same structures to preserve and publish semantic-rich data and equally consume data from other sources. Ontologies provide a common medium for inter-agent information exchange, interoperation and integration. As ontologies specify the semantics of terms at the conceptual level based on the explicit conceptualization of a domain, they are understandable and easily processed by both humans and machines, thus increasing the potential of automation.

\subsection{Related work}

Ontology-based modelling has been extensively explored in the domain of context modelling [17, 18, 19]. This strand of work concentrates on the modelling of high-level abstract contextual concepts and/or facts, such as person, location, activity and computational entity, either sensed, static or profiled, with constraints and annotations. While it provides some general guidance as a type of upper-level ontology, it fails to capture specific characteristics of SHs. Already, there have been several attempts to use ontologies to model context in an assistive living scenario [20, 21]. The use of ontologies is primarily restricted to specific pervasive aspects and usually for reasoning purposes only.

Using ontologies for SH modelling can be viewed as a recent development. Latfi et al. [22] proposed an ontological architecture for a Telehealth Smart Home (TSH) and developed prototype ontologies. Klein et al. [23] proposed a context ontology for ambient middleware as part of the European Union funded SOPRANO project [24], They claimed that ontologies will be used as a central reference document for SOPRANO middleware. Both works are at concept level. It is not clear how large-scale semantic content is created and used in real world scenarios.

While our research shares some consensus with [22] in ontology modelling and with [24] in the role and use of ontologies, it is fundamentally different from these works in that we take a broad, integrated and systematic view towards SSH. In this case, ontology-based semantic modeling and 
representation is not just used for separate, stand-alone components for some specific purposes. Rather, ontologies are regarded as a conceptual backbone and a common vehicle for enabling and supporting communication, interaction, interoperability, integration and reuse among devices, environments, inhabitants and external sources. Our focus is on how to capture and model rich semantic metadata with the emphasis being placed on the effective use and reuse of intelligent content for supporting assistive living. We also address issues pertaining to semantic data lifecycle management, namely modeling, creation, storage, use/reuse and maintenance.

There are some ongoing initiatives such as European Union projects ASK-IT [25] and SAPHIRE [26], which intend to use Semantic Web technologies for interoperability and integration. Until now the SSH concept, and in particular, the idea of using ontologies as the conceptual backbone for integration, interoperability and high-level intelligent processing, has not been witnessed.

\section{The conceptual architecture}

We propose a layered conceptual architecture for the SSH, as shown in Figure 1. The Physical Layer consists of physical hardware such as sensors, actuators, and various devices including medical equipment, household appliances and network components. This layer provides the means to monitor and capture the events and actions in a $\mathrm{SH}$, and subsequently traverse data to the Data Layer. The Data Layer archives collected raw data in a number of data stores. These stores are usually disparate in data formats and access interfaces, with each of them being dedicated to individual sensor based application scenarios. The Application Layer contains various capabilities, tools and (sub)systems for assistive living. Within this layer applications process sensory data which has been passed via the Data Layer and can be used to control actuators and/or multimedia facilities in the Physical Layer to offer assistive living as required. These three layers have so far been the core conceptual components underpinning SH application design and development. While each layer is indispensable for any $\mathrm{SH}$ application, the close coupling among sensors, data and applications, often having one to one, ad hoc relationships, causes many challenges as discussed in the introduction.

The SSH addresses these challenges by incorporating a Semantic Layer, a RDF (Resource Description Framework ${ }^{1}$ ) Data Bus and an Intelligent Service Layer in the proposed systems architecture. These layers break down the direct links between the Data and Application Layers and provide underpinning technologies for data sharing, reuse and application development. The goal of the Semantic Layer is to provide a homogeneous view over heterogeneous data, thus enabling seamless data access, sharing, integration and fusion across multiple organizations. It achieves this by using $\mathrm{SH}$ ontologies as a unified conceptual backbone for data modeling and representation. Semantic modeling allows the markup of various data with rich metadata and semantics to generate semantic content. Multiple SHs in geographically distributed locations supported by various organizations can then aggregate and fuse their SH data. No matter if the data are archived in a centralized repository or in each institution's individual repository as shown in Figure 1 the uniform data models and representation, e.g. RDF or Web Ontology Language (OWL), allow seamless data access through the RDF Bus based on the standard communication protocol HTTP and RDF query language SPARQL. The Semantic Layer is also responsible for providing tools and APIs for semantic data retrieval and reasoning. Details will be presented in next section.

\footnotetext{
${ }^{1}$ RDF, RDFS, OWL, HTTP, SPARQL and URI that will be mentioned later are all W3C standards. Detailed information can be found at W3C web site - www.w3.org.
} 


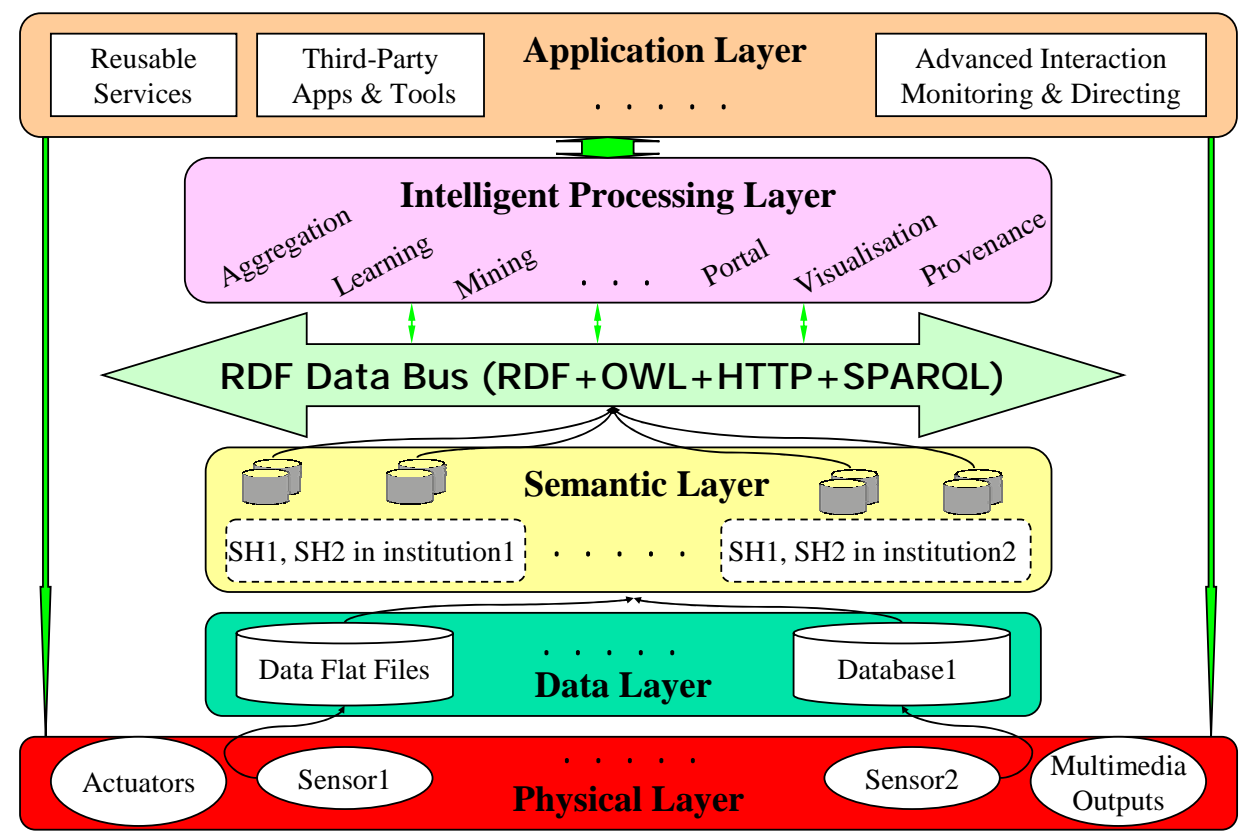

Figure 1. The conceptual architecture of the SSH

The Intelligent Service Layer is built upon the semantic content and functionalities of the Semantic Layer. Its purpose is to exploit semantics and descriptive knowledge to provide advanced processing and presentation capabilities and services. The former provides addedvalues to the query interfaces of the RDF Bus by further searching, analysing and reasoning over recorded SH data. The latter essentially visualises the contents of the repositories and the outputs of the processing services. Table 1 lists some examples of processing and presentation services. Such a list of processing and presentation services is illustrative and not exhaustive; furthermore, it does not mean that each SH will use all these services. In fact the selection and use of such services will depend on the nature and availability of collected data as well as the personal needs of inhabitants and care providers, hence exploiting further the concepts of personalisation. These services can be realized using industrial standards such as Web services [27] and are given welldefined meaning, e.g. semantic Web services [28]. They are accessible to third party developers, thus interoperable and reusable at both the service and application level.

Table 1. A list of example processing and presentation services

\begin{tabular}{|c|c|}
\hline Processing services & Presentation services \\
\hline $\begin{array}{l}\text { - } \begin{array}{l}\text { Compare Activities of Daily Living (ADL) of } \\
\text { subjects in the same group and/or different } \\
\text { groups }\end{array} \\
\text { - Aggregate multiple data sources to create a } \\
\text { single virtual large data set for data mining } \\
\text { - Offer semantic based search and discovery } \\
\text { - Extract user profiles and ADL patterns } \\
\text { - Create inhabitant communities for social or } \\
\text { - medical purposes } \\
\text { re-construct an ADL trace from a living context }\end{array}$ & $\begin{array}{l}\text { - Browsing and navigation facilities over a single } \\
\text { or federated SH repositories } \\
\text { - Visualise ADL and their differences of subjects } \\
\text { in the same group and/or different groups } \\
\text { - Illustrate relationships of ADL from a more } \\
\text { semantic viewpoint } \\
\text { - Present graphically the results of various } \\
\text { statistical and probabilistic analysis in mining } \\
\text { and learning } \\
\text { Allow professional carers to specify } \\
\text { inhabitants' ADLs in a graphical manner }\end{array}$ \\
\hline
\end{tabular}


The Semantic Layer essentially achieves data interoperability and machine understandability, whereas the Intelligent Service Layer delivers the capability of interoperability and high level automation. As such, the proposed architecture enables a novel and flexible paradigm of SH system development and deployment. In this paradigm services in the Intelligent Service Layer are responsible for data access and the provision of processing and presentation capabilities. They have well-defined interfaces and can be boxed as primitive off-of-shelf building blocks. SH systems shall have little direct interaction with data at the lower layers. SH system development will be accomplished by the aggregation and assembling of various on-demand services in terms of SH system requirements. New functionalities, i.e. capabilities and services, can be made available whenever needed. Eventually a robust feature-rich technological infrastructure will be in place to facilitate the delivery and agile deployment of assistive living solutions, e.g. plug and play and open system development.

\section{Semantic modelling and content management}

This section describes semantic modeling, semantic content creation and manipulation - the key enabler for the proposed approach. Figure 2 depicts the core components and technologies in which ontologies are used as commonly agreed uniform data models to imbue raw data from various data sources with rich metadata and semantics. Both ontologies and generated semantic content are represented using expressive Web ontology languages such as RDF or OWL and stored in data repositories in which all data are semantically interlinked. Semantic content can be understood and processed by machines or agents, thus allowing a high level of automation, seamless data access, retrieval and reasoning.

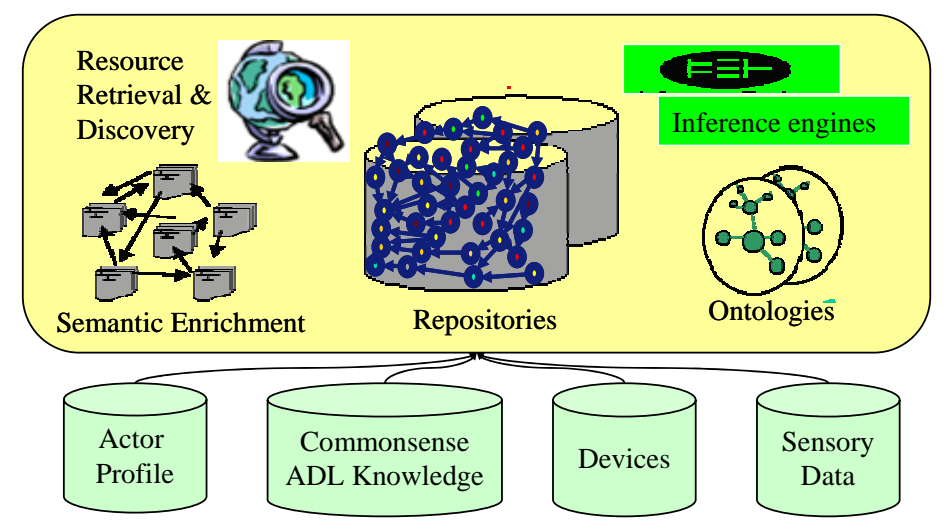

Figure 2. The core components for semantic management

\subsection{Semantics, semantic modelling and representation}

Semantics refer to meaning. Semantic modelling refers to the process of defining the meaning of data, devices and services. The basic formalism used for semantic modelling is the RDF. RDF is a graph data model for objects ("resources") and relations between them. An RDF graph contains a set of triples, each triple consisting of the subject, predicate (property) and object. This structure can be considered as a natural way to describe the vast majority of the data processed by machines. A triple can make assertions that particular things (such as sensors, inhabitants) have properties (such as "is used for", "has a type") with certain values (thermostat, dementia). Figure 3 shows the RDF graph that represents the group of statements "there is a Contact Sensor 
identified by http://www.ulster.ac.uk/ssh2008/ssh\#Trail_lab_contact_sensor_9, and it is located in the second cupboard kitchen_cupoard_2 of the kitchen, attached to the bottle milkBottle of milk and it activates the milk_moved event.

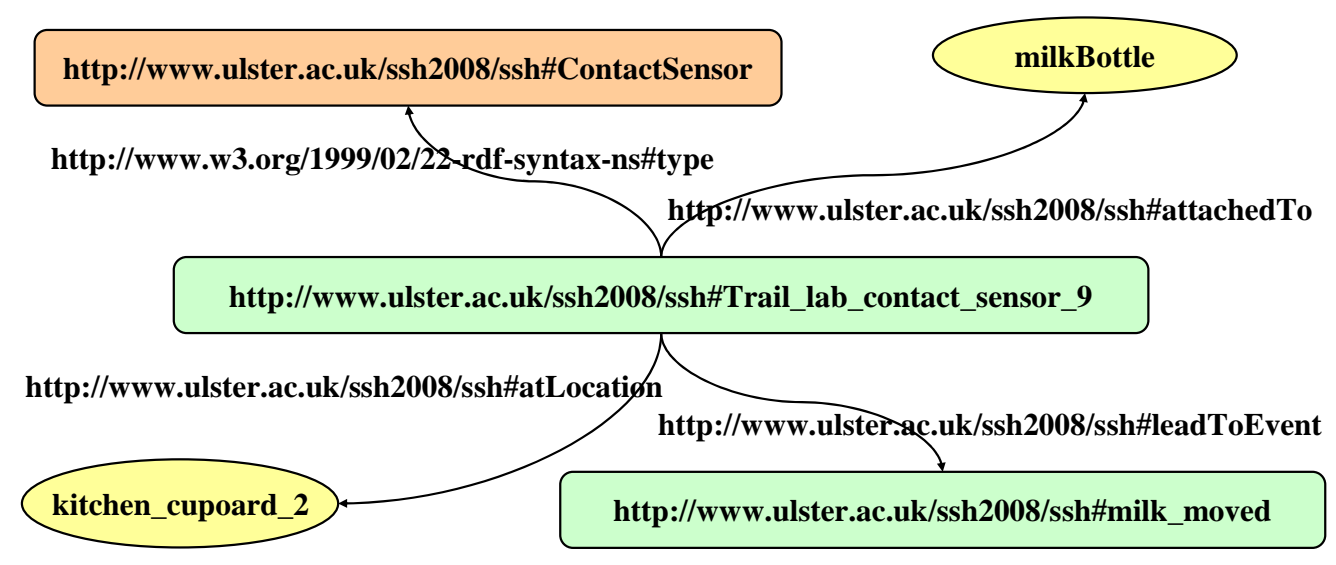

Figure 3. An RDF graph describing a concrete contact sensor

RDF encodes meaning in their triple statements. A single separate data item does not stand for anything. Its meaning can only be interpreted against a context in which the term appears. In an RDF expression, the subject, predicate and object are all identified by a Universal Resource Identifier (URI) - see Figure 3. This ensures that concepts and properties are not just terms (keywords) in a domain but can be tied to a context where their unique definitions can be interpreted. The context is in essence the ontologies that formally define all core concepts and the relations between them. A typical ontology usually contains a hierarchical structure of concepts and subconcepts. Relations between concepts are established by assigning properties to concepts and allowing subconcepts to inherit such properties from their parent concepts. In the above example the ontology is defined in http://www.ulster.ac.uk/ssh2008/ssh.

Semantic representation is accomplished through ontology languages such as the RDF Schema (RDFS) and OWL. RDFS defines a vocabulary (terms) for describing the properties and classes of RDF resources, with semantics for generalization hierarchies of such properties and classes. Figure 4 is the RDF representation of the RDF graph in Figure 3. On top of RDFS OWL adds more vocabulary for describing properties and classes: among others, relations between classes (e.g. disjointness), cardinality (e.g. "exactly one"), equality, richer typing of properties, characteristics of properties (e.g. symmetry), and enumerated classes. This gives OWL more expressive power for representing complex data semantics.

Ontology languages carry built-in inference rules from underlying data models such as RDF graphs or OWL's Description Logics. This subsequently gives semantic representation further power to enable inference and reasoning via the notion of entailments. An ontology may express the rule "If an inhabitant's action is in response to an event, and a sensor generates that event from one change, then the inhabitant's action can be associated with that change". A program could then deduce, for instance, that the action is the direct reaction to the change. The computer doesn't truly "understand" any of this information, however, it can now manipulate the terms much more effectively in ways that are useful and meaningful to human users. 


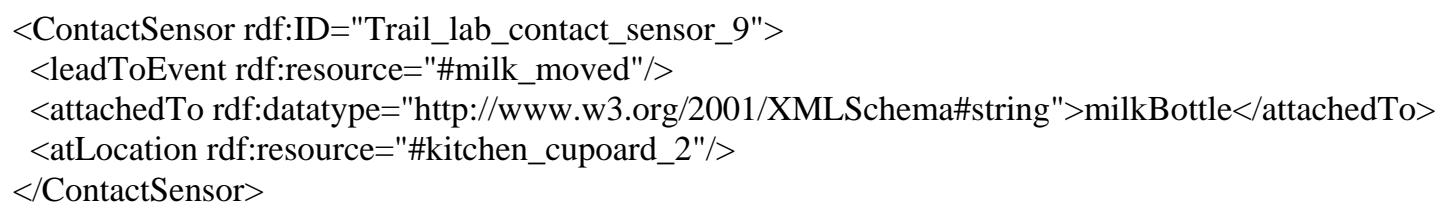

Figure 4. The RDF representation of the RDF graph in Figure 3

\subsection{Smart home ontology engineering}

Ontology development is a formal process of knowledge acquisition and modelling. It requires the close cooperation of domain experts and knowledge workers. Domain experts identify and describe concepts, properties, their relations, instances and role-playing actors within a problem domain, and domain-specific, application-dependent problem solving processes. Knowledge workers, who do not have domain background, will use extensive knowledge engineering techniques to capture useful knowledge based on the experts' expertise and will develop knowledge-preserving structures, i.e. models, which can hold and share reusable information. As such, domain analysis and characterisation is essentially the first step of ontology development.

A SH is a complex micro-ecosystem that usually consists of the following constituents:

(1) a physical environment with various pieces of furniture, electrical/electronic household appliances, and rooms which provide a living space,

(2) inhabitants that perform various activities within the environment,

(3) sensors, actuators and medical devices to sense and act on environmental changes and inhabitant behaviors,

(4) assistive resources including actors (care-providers or family members), middleware services or applications to respond to events and situations.

Each of these constituents plays an indispensable role and provides specific functions. Overall they deliver 'just-in-time' assistance for inhabitants through inter-communication and causal interactions.

Based on the above characterization a SH can be modeled in seven ontologies. They include an ontology for the physical equipment such as sensors, actuators, medical devices and home electronic or electrical appliances; an ontology for actions and ADLs such as watching television and making drinks; an ontology for living spaces and environments such as the kitchen, sitting rooms; an ontology for actors such as inhabitants, care-providers; an ontology for medical information; an ontology for software components such as services and applications and an ontology for time in order to model temporal information. Each ontology is used to explicitly conceptualise a specific aspect and overall they provide a semantic model for smart homes. Figure 5 shows some classes and properties of SH ontologies which have been developed using the Protégé tool [29]. It is worth noting that existing well-defined ontologies could be imported and reused directly, for example some medical ontologies and a time based ontology [30].

\subsection{Semantic content creation}

Ontologies are knowledge models in which a concept is a structure for preserving knowledge. An instantiated concept, referred to as an instance, is a concrete piece of knowledge. Thus semantic content creation is equivalent to instance generation, which is achieved by describing the raw data source using pre-defined ontologies, i.e., the so-called semantic annotation. 
There are two major approaches to conducting semantic annotation. One is to use generic ontology editing tools such as the Protégé OWL Plugin [29]. These tools can usually be used to perform several activities in one go, such as knowledge acquisition, ontology editing, knowledge population as well as knowledge base creation. They are feature rich but require professional knowledge engineering expertise. So this method is suitable for knowledge engineers. Another approach is to develop domain specific dedicated lightweight annotation tools for domain experts or resource (data) providers to carry out semantic annotation and create knowledge repositories. Such tools are often designed to provide intelligent semi(automatic) support for knowledge acquisition and modeling, including automated information extraction, classification and completion, to help create instances.

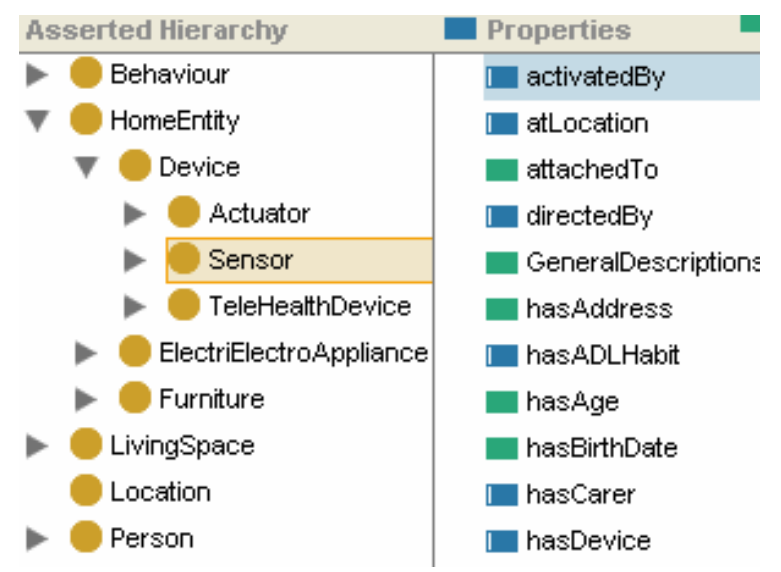

Figure 5: Some classes and properties of the SH ontology

Given the nature of data in SHs we propose a two phase semi-automatic approach to semantic descriptions. In the first phase data sources such as sensors and devices are manually semantically described. As the number of data sources in a $\mathrm{SH}$ is relatively limited, though large, it is manageable to create all semantic instances manually by generic ontology editors such as the Protégé OWL Plugin. Figure 6 shows an instance of SSH inhabitant that is created in the Protege and represented in OWL. In the second phase dynamically collected sensory data are first converted to textual descriptors. For example, a contact sensor returns a two-state binary value. It can be pre-processed to literals sensible for denoting two states such as on/off or open/close or used/unused, etc. The concrete interpretation of the state depends on the purpose of the sensor. For example, the two states of a contact sensor in a microwave could be open/close. If the contact sensor is attached to a milk bottle, the literal might be used or unused. The conversion of numerical values to descriptive terms is to facilitate interpretation and comprehension for both humans and machines. Pre-processed data can then be automatically attached to semantic instances of the corresponding data source to create a data repository.

\subsection{Semantic content storage and retrieval}

Once semantic data are generated, they can be archived in semantic repositories for later exchange or consumption by various applications (e,g, mining and integration). Semantic repositories are essentially knowledge bases consisting of millions of RDF triples. They are built on top of traditional database management systems by adding a semantic processing layer for semantic manipulation. Several semantic repository technologies such as [31,32] are available, which could be inspiring and motivating for SSH. 
Repositories may be centralized in one location or distributed in geographically dispersed sites. As all repositories share the same model, i.e. ontologies, and often use the same type of access APIs, there is little difference in the retrieval of semantic data. Nonetheless, distributed repositories are required to deal with issues pertaining to security and communication bandwidth. Within SH based assistive living, data may be exchanged and shared between institutions in different countries at a global scale. It would be desirable for each institution to have a repository and its own authorization and authentication control for the enforcement of local data usage policies and ethical issues. On the other hand, as the volume of various data in a single SH is expected to be reasonably low, a centralized repository should be cost effective and easy for management. We therefore suggest that the SSH infrastructure adopts distributed repositories at the inter-institution level and a centralised repository within an institution.

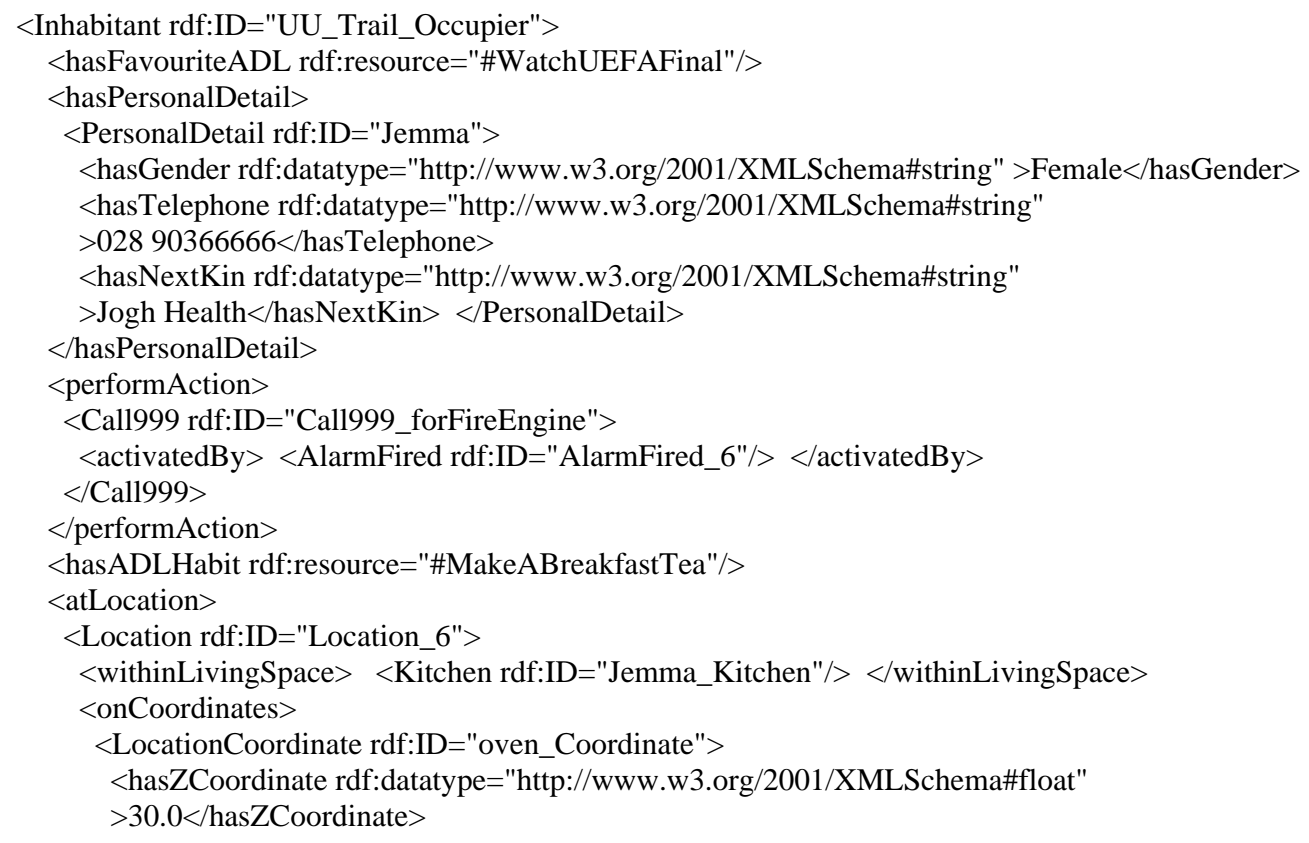

Figure 6: A fragment of the OWL representation of the inhabitant instance

A centralized repository may be conceptually divided into two interlinked components, as shown in Figure 7, based on the nature of SH data. The first component contains semantic descriptions relating to the various devices, inhabitants, individual SHs and the services offered within an institution. These entities and their semantic descriptions are relatively stable for a care institution, i.e. static data. This component can functionally serve as a registry so that new SHs once built within the institution, devices once added to any individual SH, inhabitants once they take residence in a $\mathrm{SH}$ and new services once developed can all be registered for later discovery and reuse. The second component is dedicated to the storage of dynamically generated sensory data and derived high level ADL data, which are time dependent, varying and extensive, i.e. dynamic data. Static data only need to be described and recorded once while dynamic data have the requirement to be recorded whenever they are generated. The separation of their storage saves storage space and also increases recording efficiency. Another advantage with this design is its ability to supports dynamic, automatic discovery of devices, device data, services and inhabitants, thus facilitating reuse of data and services. Further details of these concepts will be presented in the following Section. 


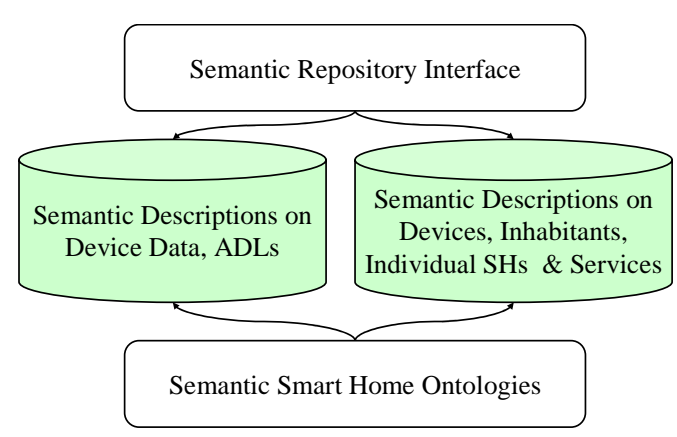

Figure 7: The Semantic Repository within the SSH

\section{Semantic enabled processing capabilities and applications}

Semantic modelling gives data many characteristics that are otherwise not available. Firstly, it enables the data to be exchangeable, interoperable and accessible at both intra- and interorganisation levels based on the commonly accepted ontological schema. Secondly it makes data understandable and easily processed by both humans and machines (or software agents). Thirdly, semantic data supports reasoning and inference by incorporating entailment rules in expressive representation. These attributes make semantic data amenable for flexible and complex manipulation, thus enabling many advanced processing capabilities such as automated processing and knowledge discovery, and novel application scenarios such as data sharing, reuse, integration, and situation aware assistance.

Given the manner in which semantic data are used is only limited by the application's requirements and the developer's imagination, it is unwise and practically impossible for us to try to elaborate all usage mechanisms. As such this section will omit discussions relating to the basic use of semantic data, for example how to facilitate data sharing and exchange, how to carry out semantic retrieval and searching, as these features have already been elaborated and illustrated through research results in various domains. Instead we shall discuss some core innovations brought specifically to SHs through the combination and synergy of these semantic data properties.

\subsection{Towards a paradigm of extensible and flexible assistance provisioning}

The SSH concept can support an open, plug-and-play paradigm that makes assistance provisioning extensible, flexible, facilitates rapid prototyping and is easy-to-deploy. This paradigm is enabled by the explicit separation and management of entities, i.e. devices, inhabitants and individual SHs, and functionalities, i.e. services and applications. It distinguishes services, i.e. high level functional components that are used as building blocks for multiple applications, from applications, i.e. systems that are used by end users, either care providers or inhabitants for providing assistance. In particular it unties the direct links between services and applications and specific devices, environments (individual SHs) and inhabitants.

As shown in Figure 8, entities in all smart homes within an institution and services (i.e. functions) that are applicable to various data are semantically described and placed in a registry. Care providers in the Central Assistance Provisioning Environment in an Institution can discover applications from the registry based on requirements of individual inhabitants. The discovered applications are then linked to sensory data and provide assistance through data processing. In this paradigm, new entities and functions, such as a new installed sensor, a new resident or a new 
function, can then be added into the registry anytime for discovery and reuse while the whole system is still working. Therefore, it supports the plug-and-play concept and makes the system extensible. Application developers can discover and reuse available services to develop new applications, and can then publish the application in the registry for further reuse. This paradigm will significantly reduce the needs for developing new services and applications when new SHs or devices are added. Equally services and applications requiring sensory data can search the registry to discover available devices that provide the required data. This paradigm saves not only effort for the development and cost for new devices, however, also facilitates rapid prototyping and easy deployment.

The above discussion is made in the context of a $\mathrm{SH}$ based care institution(s). It is actually applicable to more generic scenarios. For example, individual SHs could be geographically dispersed across a wide area without belonging to any specific care institutions. In an extreme case, ordinary family homes could be connected to such assistive systems through broadband, passing data and receiving advice. The key point we wish to make is that the SSH concept enables an open home paradigm for assistance provision.

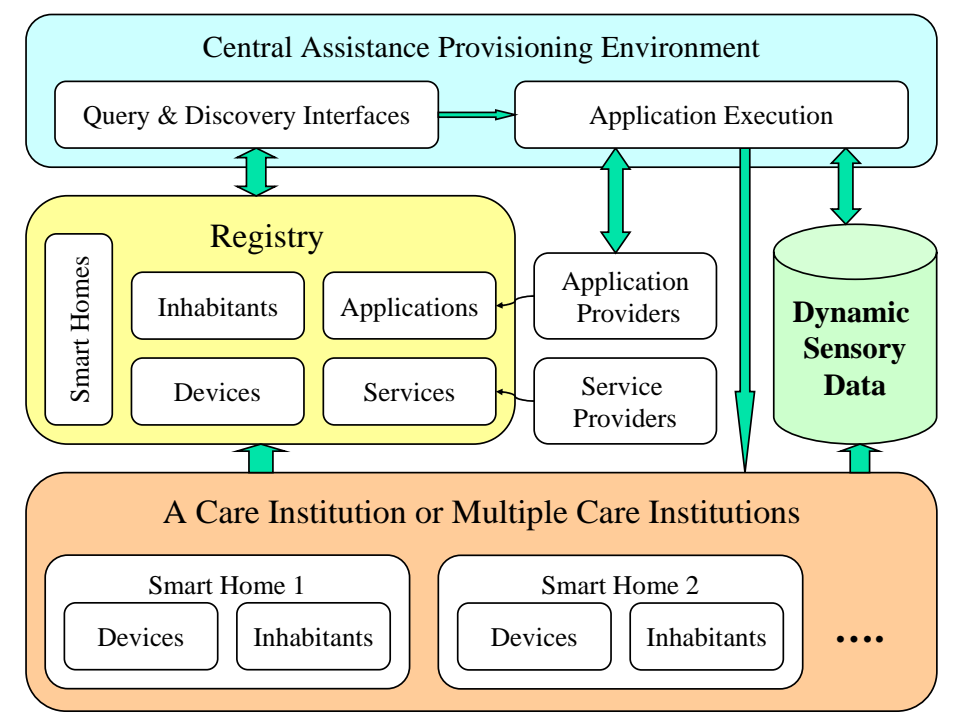

Figure 8: An open paradigm for assistance provision

\subsection{Cognitive ADL monitoring and recognition}

Current SH can provide reminding assistance through pre-defined instructions, such as the instruction to take medicines as a specific time [33], to perform ADLs such as prepare a meal [10] and reactive emergency handling, for example, calling for a fire engine when a fire breaks out. In the future, however, it is increasingly expected that assistance at a behavioral level be provided for the elderly. A particular group within this cohort who would benefit from the deployment of SH technology would be those suffering from cognitive deficiencies such as Alzheimer's disease. For these persons it then becomes necessary to monitor their behaviour and recognize their intended ADLs so that just-in-time assistance can be provided.

Semantic modeling and reasoning can achieve this in a scalable and automatic way by building ontological behavioral models. The basic idea is that through semantic modeling we can build an ADL ontology as shown in Figure 9 with each node denoting a type of ADL. Each ADL class is described with a number of properties and sub-classes can inherit all properties from its parent 
class. A property is defined by specifying its domain and range. The domain refers to all classes that can be described by the property and the range refers to all classes whose instances can be assigned to the property. A property describes a class using either a literal or an instance of another class as its value, thus linking two classes. This essentially gives rise to a description based behavioral model, i.e., an ADL is described by various properties.

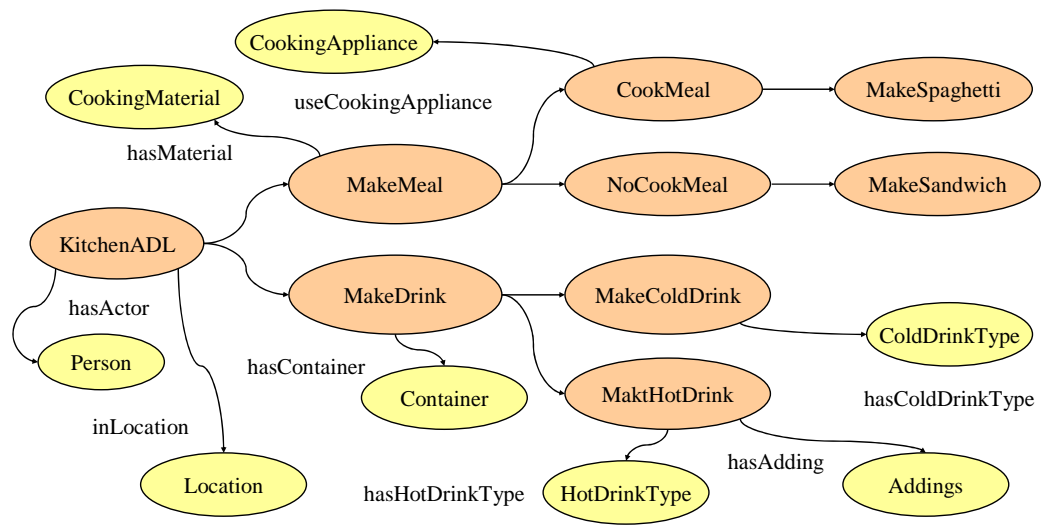

Figure 9. A fragment of the graphical hierarchy of the ADL ontology

The underlying mechanisms for ADL monitoring and recognition is straightforward and natural. If we can identify a number of properties, then we could infer and recognize an ADL or ADLs based on the described ontological behavioural model. In semantic modeling the perception of an event and/or the detection of sensory signals imply the identification of a concrete instance of a class. For example, the activation of a contact sensor in a cup means that the cup, as an instance of Container, is used in an ADL. As the Container class is the range of the hasContainer property, it can be inferred that the hasContainer property is assigned the value cup. Since the hasContainer property is used to describe the MakeDrink class, it can be further inferred that a MakeDrink ADL has taken place. Nevetheless, it is not possible to ascertain whether the ADL is MakeHotDrink or MakeColdDrink as both ADLs have the hasContainer property. This is exactly one of the advantages of the description based ADL recognition because based on limited sensory information the system can still identify uncertain high level ADLs. In the given example, though we can not tell the concrete ADL, i.e. the MakeHotDrink or the MakeColdDrink, we can at least know that the inhabitant is performing a MakeDrink ADL. When more sensory becomes available, concrete ADL(s) can be identified. Suppose that a contact sensor in a tea container is activated, this implies that an instance of the HotDrinkType class, i.e. the tea, has been specified, and the hasHotDrinkType is assigned the value tea. In this case it is reasonable to assume that an ADL or ADLs that happen, though we do not know yet, must have at least the two properties hasContainer and hasHotDrinkType. Based on the ontological ADL model we can infer that it is the MakeHotDrink ADL.

The ADL monitoring and recognition process can be summarized as follows: Sensory inputs are used to identify concrete items that have already been specified as instances of classes in $\mathrm{SH}$ ontologies. In terms of the scope of a property's range, the property that takes the identified item as its value can be inferred. In terms of the scope of a property's domain the ADL(s) that can be described by the inferred properties can then be recognized. As properties can be inherited from super-classes (higher level abstract ADLs) to sub-classes (lower level concrete ADLs), the lower a class is in the ADL class tree the more properties it has. This means that the more sensory data that are available, the more accurately ADLs can be recognized. For example, if we only have the location sensory data as inKitchen, we can only infer the inhabitant might perform a KitchenADL 
at that specific point in time, without knowing what ADL it is. If further data become available, for example cup sensory data, then we can infer the inhabitant might perform the MakeDrink ADL at that specific point in time. Nevertheless we still do not know what drink the inhabitant will make. If we obtain the coffee sensory data, then we can determine that the inhabitant is making coffee but we still do not know if it is a white coffee or a black coffee. Hence the sensory data from milk or sugar sensors can further help to recognize the details of the performed ADL. From what we have described above, it is apparent that the proposed approach can monitor the unfolding of an ADL and incrementally recognize the ultimate ADL, which may be considered as not previously possible. The monitoring and recognition process conceptually corresponds to the subsumption operation of description logic based reasoning, which can be realized using reasoners such as FaCT [34] or RACER[35].

The semantic model which enables behavior monitoring and recognition has a number of compelling advantages: Firstly, the scalability of SH ADL modeling has been a bottleneck to effective behavioral recognition. It is often the case that proof-of-concept experiments, either state-based or process-based approaches, work well but fail to scale up. Ontological ADL modeling does not have this problem with the extensive technological support which is offered in ontology engineering, which includes tools, APIs, storage and reasoners. Ontologies of thousands of classes have been developed in other domains, e.g. 7,000 concepts in the gene ontology, and semantic data repository of 25 million triples is also practiced in TripleStore [32]. For smart homes, ADL classes and associated instances are simply not present in a such scale. Secondly, semantic ADL models contain explicit rich semantics and built-in logical entailment rules. This allows not only humans but also software agents (such as assistive systems) to interpret, comprehend and reason against captured semantic ADL data. As such, behavior monitoring and recognition can be realized at higher levels of automation. Thirdly, description based reasoning provides a mechanism to incrementally predict ADLs by interpreting limited or incomplete sensor data. This capability is particularly important because assistive systems are supposed to provide reminding or suggestive assistances with limited sensory data.

\subsection{Knowledge based assistive living systems}

Ontologies are knowledge models. ADL classes and their hierarchical structure in SSH ontologies are in essence the explicit model and representation of the commonsense knowledge of a human's daily activities and their classification. In addition to these generic ADLs, individual inhabitants have their own living habits, regular ADL patterns, preferences and unique ways to respond to various events. Such individual lifestyles may further vary with age profile, culture and personality. Using semantic modelling we can formally capture, model and represent an individual inhabitant's personal specialties in semantic repositories. These heuristics and knowledge can then be exploited for intelligent living assistance. A typical example is personalised assistance. Consider that a MakeDrink ADL is recognised as described in Section 5.2 for a person with dementia. An example of general assistance provided would be to advise the person to make either a cold drink or a hot drink. Then the assistive system will monitor the person's behaviour and advise its actions accordingly. If the person's preferences on making a drink are known, e.g., she/he likes hot white coffee, then the assistive system can directly remind the person what she/he should do in order to make their coffee hot with milk. Similar assistance can be offered for recommending other ADLs, for example TV channels, etc.

Another knowledge based use scenario is adaptive assistance. Rather than modelling an inhabitant's behavioural preferences a priori, an assistive system can derive an inhabitant's ADL patterns through data mining and pattern recognition against collected semantic data. This will capture the evolution of an inhabitant's daily life and incorporate changes into behavioural 
models. An assistive system can then reason against learnt ADL patterns to provide adaptive assistances.

\section{Conclusions and future work}

Research on SHs and assistive living has come to a critical point, i.e. novel paradigms and technologies are needed in order for the approach to be useful in real world scenarios in terms of applicability, scalability and ease of use. This paper has introduced the concepts of SSHs that aims to break down barriers (heterogeneity) and isolations (hardwired) among devices, data, capabilities and applications, and to unleash the potential of the approach through semantics, rules and expressive representation. We have proposed an integrated systems architecture for SSHs and discussed its core functional components and interplay. We have described in detail the methodology and related technologies for semantic modelling and semantic content management. Though semantic content could be used in many different ways for many purposes, we have concentrated on the elaboration of a semantic enabled paradigm for assistance provisioning and three exemplar use scenarios due to space limitation have been presented.

The SSH concept, its enabled assistive living paradigm and underpinning technologies await further investigation, development and evaluation through real world use case studies. Nevertheless, our work has laid a solid architectural and methodological foundation. Initial results have demonstrated the potential and value of the approach and further clarify future research directions. We believe that SSHs are the next generation of technological infrastructures for assistive living that facilitates the innovative exploitation of research results from AI, Web technologies and information processing.

\section{References:}

1. Center for Future Health, University of Rochester, Smart Medical Home Research Laboratory, June 12, 2007 http://www.futurehealth.rochester.edu/smart_home

2. MIT Department if Architecture, House_n “The PlaceLab”, June 12, 2007, http://architecture.mit.edu/house_n/placelab.html

3. Georgia Institute of Technology, "The Aware Home," June 12, 2007, http://www.cc.gatech.edu/fce/ahri/

4. Dittmar, A., Axisa, F., Delhomme, G., Gehin, C.: New concepts and technologies in home care and ambulatory monitoring. In A. Lymberis and D de Rossi (eds) Wearable eHealth Systems for Personalised Health Management: State of the Art and Future Challenges, IOS Press (2004) 935.

5. Helal, S., Mann, W., El-Zabadani, H., King, J., Kaddoura, Y., Jansen, E.: The gator tech smart house: a programmable pervasive space. IEEE Computer (2005) 64-74.

6. Alive Technologies, http://www.alivetec.com

7. PhMon Personal Health Monitoring System with Microsystem Sensor Technology http://www.phmon.de/englisch/index.html

8. OSGi - The dynamic module system for Java, http://ww.osgi.org

9. Konnex - Open standard for home and building control, http://ww.konnex.org

10. Philipose, M., Fishkin, K.P., Perkowitz, M., Patterson, D.J., Fox, D., Kautz, H., Hahnel, D.: Inferring activities from interactions with objects. IEEE Pervasive Computing(2004) 50-57

11. Bouchard, B., Giroux, S., Bouzouane, A.: A logical approach to ADL recognition foralzheimer's patients. In: Proc. of the 4th International Conference on Smart Homes and Health Telematic (ICOST’06). (2006) 1-8 
12. Hong X., Nugent C., Mulvenna M., McClean S., Scotney B. and Devlin S. (2008) Evidential fusion of sensor data for activity recognition in smart homes, Pervasive and Mobile Computing, 2008 (in press).

13. Davies J., Studer R. and Warren P. (2006) Semantic Web Technologies: Trends and Research in Ontology-based Systems, Wiley, ISBN: 978-0470025963

14. Pollack M. Intelligent technology for an aging population: the use of AI to assist elders with cognitive impairment.” In AI Magazine, vol.26, no.2, pp.9-24, 2005.

15. Staab S. and Studer R. (2004) Handbook on Ontologies (International Handbooks on Information Systems), Springer ISBN: 978-3540408345

16. Davies J., Fensel D., van Harmelen F. (2003) Towards the Semantic Web: Ontology-Driven Knowledge Management, Wiley, ISBN: 978-0470848678

17. Strang, T., Linnho.-Popien, C., Frank, K.: CoOL: A Context Ontology Language to enable Contextual Interoperability. In Stefani, J.B., Dameure, I., Hagimont,D., eds.: LNCS 2893, Springer Verlag (2003), pp.236-247

18. Chen, H., Finin, T., Joshi, A.: An Ontology for Context-Aware Pervasive Computing Environments. Special Issue on Ontologies for Distributed Systems, Knowledge Engineering Review (2003)

19. Gu, T., Wang, X.H., Pung, H.K., Zhang, D.Q.: An Ontology-based Context Model in Intelligent Environments. In Proceedings of Communication Networks and Distributed Systems Modeling and Simulation Conference, San Diego, California, USA (2004)

20. Preuveneers, D., Van den Bergh, J., Wagelaar, D., Georges, A., Rigole. P., Clerckx, T., Berbers, Y., Coninx, K., Jonckers, V., De Bosschere, K. (2004) Towards an Extensible Context Ontology for Ambient Intelligence. Proc. of EUSAI 2004, Springer, LNCS vol. 3294, 148-159

21. Roussaki, I., Strimpakou, M., Pils, C., Kalatzis, N., Anagnostou, M. Hybrid context modelling: A location-based scheme using ontologies. Proceedings of the Fourth Annual IEEE International Conference on Pervasive Computing and Communications Workshops (PERCOMW'06) (2006)

22. Latfi, F., Lefebvre, B., Descheneaux C. (2007) Ontology-Based Management of the Telehealth Smart Home, Dedicated to Elderly in Loss of Cognitive Autonomy, Proceedings of the OWLED 2007 Workshop on OWL: Experiences and Directions, http://www.webont.org/owled/2007/PapersPDF/submission_37.pdf

23. Klein, M., Schmidt, A. and Lauer, R., (2007) Ontology-Centred Design of an Ambient Middleware for Assisted Living: The Case of SOPRANO, In proceeding of Towards Ambient Intelligence: Methods for Cooperating Ensembles in Ubiquitous Environments (AIM-CU), 30th Annual German Conference on Artificial Intelligence (KI 2007), Osnabrück, 2007

24. The EU SOPRANO IP project http://www.soprano-ip.org/

25. The EU ASK-IT IP project, http://www.ask-it.org/

26. The EU SAPHIRE project http://www.srdc.metu.edu.tr/webpage/projects/saphire/index.php

27. Chinnici, R., Gudgin, M., Moreau, J. and Weerawarana, S. (2002) Web Services Description Language (WSDL) 1.2, W3C Working Draft, 9 July 2002. http://www.w3.org/TR/wsdl12/.

28. W. Johnston, "Semantic Services for Grid-Based, Large-Scale Science," IEEE Intelligent Systems, vol.19, no.1, pp.34-39, 2004.

29. The Protégé, http://protege.stanford.edu/

30. The time ontology in OWL, http://www.w3.org/TR/owl-time/

31. Horrocks I., Li L., Turi D. and Bechhofer S., The instance store: DL reasoning with large numbers of individuals, Proc. 2004 Description Logic Workshop, pp.31-40, (2004)

32. Harris S. and Gibbins N., 3store: Efficient Bulk RDF Storage, Proc. 1st International Workshop on Practical and Scalable Semantic Systems (PSSS'03), pp.1-15, (2003)

33. Nugent, C.D., Finlay, D.D., Davies, R.J., Mulvenna, M.D., Wallace, J.G., Paggetti, C., Tamburini, E. and Black, N.D., The next generation of mobile medication management solutions, International Journal of Electronic Healthcare, vol.3, no.1, pp.7-31, (2007) 
34. Horrocks I., Sattler U. and Tobies S., Practical reasoning for expressive description logics, Lecture Notes in Artificial Intelligence, No.1705, H. Ganzinger, D. McAllester, and A. Voronkov, eds, pp.161-180, (1999)

35. Haarslev V. and Möller R., Racer: A Core Inference Engine for the Semantic Web, Proc. 2nd International Workshop on Evaluation of Ontology-based Tools (EON2003), ISWC 2003, pp. 2736, (2003) 